\title{
A kind of vehicle millimeter wave SAR system
}

\author{
Kai-Bo Cui ${ }^{\dagger}$, Xi Chen, Jing-Jian Huang and Nai-Chang Yuan \\ College of Electronic Science and Engineering, \\ National University of Defense Technology \\ Deyacun, Hunan province, Changsha 410073, China \\ †E-mail: 764608294@qq.com
}

\begin{abstract}
The airborne and spaceborne SAR system are not universal access to a large area due to its higher cost and harsh demand conditions. This paper presents a vehicle millimeter wave SAR system. According to the features and application needs of the platform, this system uses range migration algorithm (RMA). Firstly, the system uses dechirping algorithm to deal with every echo. Then, we derive the two-dimensional frequency domain expression of the echo whose residual video phase (RVP) has been compensated and then analogize it to the spatial frequency domain to calculate the spatial frequency domain expression. Finally we complete the location reconstruction of the target. Due to the fan-shaped distribution of the signal distribution in the spatial frequency domain, there will be a large error if we reconstruct the location of the target directly. In this paper, we use stolt interpolation to turn the fan-shaped distribution into a rectangular distribution and the specific interpolation step is given. We also simulate the algorithm proposed in this paper. Finally, we use the vehicle millimeter wave SAR system to image for the actual scenario and the focusing effect of the image results is good to meet the demand. So the system can be applied to a variety of areas.
\end{abstract}

Keywords: SAR; Millimeter Wave; Vehicle; RMA; Stolt Interpolation.

\section{Introduction}

Synthetic Aperture Radar (SAR) is an active electromagnetic wave imaging sensors which can obtain the radar images in the target area. With the characteristic of all-time and all-weather, SAR has been widely used in the areas of monitoring the marine resources, regulating the terrain and measuring the target and so on. These applications are mainly based on airborne platform or spaceborne platform [7], whose characteristics are high grazing angle, strong target echo energy, stable platform and high movement speed. In this way, it is relatively easy to obtain the high-resolution SAR images. With the acceleration of the national construction, the regulation of rivers and lakes has become more and more important. But the higher cost of the airborne and spaceborne SAR system, especially the good landing conditions for the airborne platform causes that it is not universal access to a large area. So the development of the vehicle 
SAR system is imminent. For the vehicle platform, due to its self-limited conditions, the grazing angle is small and the movement speed is slow, which all make it more difficult to obtain high-resolution SAR images.

This paper proposes a vehicle millimeter wave SAR system and researches the high-resolution imaging algorithm. The absolute band of millimeter wave is wide and antenna beam is narrow, so its anti-jamming ability is strong [6]. Millimeter wave also has strong ability through the smoke, fog, ash and dust, so it has good weather capability. The millimeter-wave system is easy to be highly integrated due to its small size and light weight, so it is applicable to the vehicle platform.

\section{RMA and Stolt Interpolation}

As RMA reconstructs the original position of the target without any approximation, so it can be applied to a variety of situations in theory, but the subsequent interpolation operation will greatly increase the computation $[1,2,3]$. For the vehicle SAR system which demands lower in real-time, RMA is more suitable for this kind of platform.

If the radar system transmits chirp signal, the echo of a point target can be expressed as:

$$
s_{p}\left(t_{r}, t_{a}\right)=\operatorname{Arect}\left(\frac{t_{r}-\frac{2 R_{t}}{c}}{\tau}\right) e^{j 2 \pi\left(f_{c}\left(t-\frac{2 R_{t}}{c}\right)+\frac{k_{r}}{2}\left(t_{r}-\frac{2 R_{t}}{c}\right)^{2}\right)}
$$

Among them, $f_{c}$ is the signal frequency and $k_{r}$ is the modulation frequency of the chirp signal and $t_{r}$ is the range time and $t_{a}$ is the azimuth time and $t$ is the whole time which satisfies $t=t_{m}+t_{r}$ and $c$ is the speed of light and $\operatorname{rect}\left(\frac{t}{\tau}\right)$ shows a rectangular pulse whose width is $\tau$ and $A$ is a constant. $R_{t}$ is the slant distance which can be expressed as $R_{t}=\sqrt{R_{0}^{2}+\left(V_{a} t_{a}\right)^{2}}$ when in the positive side. $R_{0}$ is the original distance between the radar and the target, $V_{a}$ is the lateral movement speed of the radar.

In order to reduce the amount of data of the system, we firstly use dechirping algorithm to process the echo signal, which uses a reference signal with a fixed reference distance and the same frequency and modulation frequency of the transmitted signal to do the difference frequency treatment. If the reference distance is $R_{r e f}$, the dechirping result is : 


$$
\begin{aligned}
& s_{d}\left(t_{r}, t_{m}\right)= \\
& \operatorname{Arect}\left(\frac{t_{r}-\frac{2 R_{t}}{c}}{\tau}\right) e^{-j \frac{4 \pi}{\lambda} R_{t}} e^{-j \frac{4 \pi k_{r}}{c}\left(t_{r}-\frac{2 R_{r e f}}{c}\right)\left(R_{t}-R_{r e f}\right)} e^{j \frac{4 \pi}{\lambda} R_{r e f}} e^{j \frac{4 \pi k_{r}}{c^{2}}\left(R_{t}-R_{r e f}\right)^{2}}
\end{aligned}
$$

In the above formula, the fourth phase term is a residual phase introduced by the dechirping process which is called the residual video phase (RVP) and we must compensate RVP in the subsequent process to avoid the image defocus.

We can perform the Fourier transform in the range direction based on principle of stationary phase. The range frequency domain expression is as the follow formula and $A_{1}$ is the constant term. We can use a correction factor $e^{-j \pi f_{r}^{2} / k_{r}}$ to multiply the follow formula to complete the compensation of RVP.

$$
S_{d}\left(f_{r}, t_{m}\right)=A_{1} \tau \sin c\left(\tau\left(f_{r}+\frac{2 k_{r}\left(R_{t}-R_{r e f}\right)}{c}\right)\right) e^{-j \frac{4 \pi\left(R_{t}-R_{r e f}\right)^{2}}{\lambda}} e^{j \frac{\pi f_{r}^{2}}{k_{r}}}
$$

For the range frequency domain signal, we also can perform the Fourier transform in the azimuth direction based on principle of stationary phase in the same way to obtain the two dimension frequency domain signal expression as follows. $A_{2}$ is the constant and $W_{r}\left(f_{r}\right)$ is the range spectral envelope and $W_{a}\left(f_{a}\right)$ is the azimuth spectral envelope.

$$
S_{d}\left(f_{r}, f_{a}\right)=A_{2} W_{r}\left(f_{r}\right) W_{a}\left(f_{a}\right) e^{-j \frac{\pi f_{r}^{2}}{k_{r}}} e^{-j \frac{4 \pi R_{0}\left(f_{c}+f_{r}\right)}{c} \sqrt{1-\frac{c^{2} f_{a}^{2}}{4 V_{a}^{2}\left(f_{c}+f_{r}\right)^{2}}}}
$$

So far we get the two dimension spectrum of the echo after dechirping process . Now, we deduce RMA for reconstructing the original position of the target. We ignore the concept of wavenumber and analyze the algorithm directly [1]. The wavenumber of a target can be expressed as follows and $K$ is the wavenumber.

$$
K=\frac{4 \pi f_{c}}{c}
$$

Considering the single-frequency signal, the fundamental frequency echo for $n$ point targets is:

$$
s\left(t_{a}\right)=\sum \sigma_{n} e^{-j \frac{4 \pi f_{c}}{c} R_{n}\left(t_{a}\right)}
$$

If we use the radar position $x$ as the independent variable, the above formula can be showed as:

$$
g(x)=\sum \sigma_{n} e^{-j \frac{4 \pi f_{c}}{c} R_{n}(x)}=\sum \sigma_{n} e^{-j K_{R_{c}} R_{n}(x)}
$$


In the formula, $R_{n}(x)$ is the distance between the radar and the target. Assuming $R_{s}$ is the distance of the scene center, so $R_{n}(x)=\sqrt{R_{s}+\left(X_{n}-x\right)^{2}}$.

We can combine $s\left(t_{a}\right)$ with $g(x)$ to obtain: $g(x)=s\left(\frac{x}{V_{a}}\right)$.

We obtained the echo Doppler spectrum by changing the echo from time domain to the Doppler domain above, in the same way, we can change the echo from the wavenumber domain to the spatial frequency domain to obtain the wavenumber spectrum. So:

$$
G\left(K_{x}\right)=\left|V_{a}\right| S\left(\frac{K_{x}}{2 \pi} V_{a}\right)
$$

Thus, we can use $K_{x} V_{a} / 2 \pi$ to take the place of $f_{a}$ in the echo frequencydomain expression and $\theta_{B W}$ represents the beam width. In this way, we can obtain:

$$
G\left(K_{x}, K_{R c}\right)=\sum \sigma_{n} e^{-j \sqrt{K_{R_{c}}{ }^{2}-K_{x}{ }^{2}} R_{s}-j K_{x} X_{n}}, K_{x} \in\left[-\frac{4 \pi}{\lambda} \sin \frac{\theta_{B W}}{2}, \frac{4 \pi}{\lambda} \sin \frac{\theta_{B W}}{2}\right]
$$

If the radar transmits the chirp signal and the echo has been processed by the dechirping algorithm. Also, RVP has been filtered, so the echo expression can be written as:

$$
s_{p}\left(t_{r}, t_{m}\right)=\operatorname{Arect}\left(\frac{t_{r}-\frac{2 R_{s}}{c}}{\tau}\right) e^{-j \frac{4 \pi k_{r}}{c}\left(\frac{f_{c}}{k_{r}}+t_{r}-\frac{2 R_{s}}{c}\right)\left(R_{t}-R_{s}\right)}
$$

For the chirp signal, its frequency has a one to one relationship with the range time, namely:

$$
f=f_{c} \pm k_{r}\left(t_{r}-\frac{2 R_{s}}{c}\right)
$$

So:

$$
\begin{gathered}
K_{R}=\frac{4 \pi f}{c}=\frac{4 \pi k_{r}}{c}\left(\frac{f_{c}}{k_{r}}+\left(t_{r}-\frac{2 R_{s}}{c}\right)\right) \\
s_{p}\left(x, K_{R}\right)=A e^{-j K_{R}\left(\sqrt{R_{s}^{2}+\left(X_{n}-x\right)^{2}}-R_{s}\right)}, K_{R} \in\left[\frac{4 \pi}{c}\left(f_{c}-\frac{k_{r} \tau}{2}\right) \cdot \frac{4 \pi}{c}\left(f_{c}+\frac{k_{r} \tau}{2}\right)\right]
\end{gathered}
$$

We can change the above formula to the spatial frequency domain to obtain: 


$$
\begin{aligned}
& S_{p}\left(K_{x}, K_{R}\right)= \\
& A_{1} e^{j K_{R} R_{s}} e^{-j \sqrt{K_{R}{ }^{2}-K_{x}^{2}} R_{s}-j K_{x} X_{n}}, K_{R} \in\left[\frac{4 \pi}{c}\left(f_{c}-\frac{k_{r} \tau}{2}\right) \cdot \frac{4 \pi}{c}\left(f_{c}+\frac{k_{r} \tau}{2}\right)\right]
\end{aligned}
$$

Firstly, we filter the high-order phase terms and the filter function is $H_{1}=e^{j \sqrt{K_{R}^{2}-K_{x}^{2}} R_{s}}$. Then, we remove the additional phase term to avoid the shift of the target position and the removal function is $H_{2}=e^{-j K_{R} R_{s}}$.

Finally, the signal becomes:

$$
S_{p}\left(K_{x}, K_{R}\right)=A_{1} e^{-j K_{x} X_{n}}
$$

After an inverse Fourier transform, we can reconstruct the geometric position of the target.

For $K_{R}$ in Eq.18, it satisfies $K_{R}{ }^{2}=K_{x}{ }^{2}+K_{y}{ }^{2}$. As $K_{R}=4 \pi\left(f_{c}+f_{r}\right) / c$ and $K_{x}=K_{R c} \sin \theta=2 \pi f_{a} / V_{a}$, so the distribution of the signal in $K_{R}-K_{x}$ plane is a rectangular distribution and the distribution of the signal in $K_{x}-K_{y}$ plane is a sector distribution. But we must carry through the two-dimensional inverse Fourier transform to reconstruct the geometric position of the target in $K_{x}-K_{y}$ plane which need the signal in a rectangular distribution. So we need to interpolate to convert the sector distribution to the rectangular distribution which is called stolt interpolation [4]. The specific steps are as follows:

1) Firstly, we define $K_{y_{s}}$ as the wave number interpolated in range direction and solve its maximum and minimum value based on $K_{R}$, namely:

$$
\begin{aligned}
& K_{y_{s} \max }=K_{R \max }+k \\
& K_{y_{s} \min }=K_{R \text { min }}-k
\end{aligned}
$$

Among them, $K_{y_{s} \max }$ is the maximum value of $K_{y_{s}}$ and $K_{R \max }$ is the maximum value of $K_{R}$ and $K_{y_{s} \min }$ is the minimum value of $K_{y_{s}}$ and $K_{R \min }$ is the minimum value of $K_{R}$ and $k$ is a constant which depends on the actual situation when imaging.

2) Then, we can solve the range data points after interpolation:

$$
\operatorname{Num}_{r}=\left\lceil\frac{K_{y_{s} \max }-K_{y_{s} \min }}{\Delta K_{R}}\right\rceil
$$

Among them, $\mathrm{Num}_{r}$ is the range data points after interpolation and \lceil\rceil represents that the value is rounded up. 
3) For the range data of the first azimuth direction, we can obtain $K_{y}$ based on $K_{R}$ and $K_{x}$. Then we can obtain its maximum value $K_{y \max }$ and minimum value $K_{y \min }$. Their corresponding positions are respectively pos $_{\max }$ and pos $_{\text {min }}$.

4) We can proceed the interpolation for the original data based on $K_{y} \rightarrow K_{y_{s}}$ and the data of $K_{y_{s}}$ is from $\operatorname{pos}_{\min }$ to $\operatorname{pos}_{\max }$.

5) Finally, we can traverse in all azimuth direction to complete the interpolation process.

In conclusion, the whole algorithm flow [5] is shown in Fig. 1.

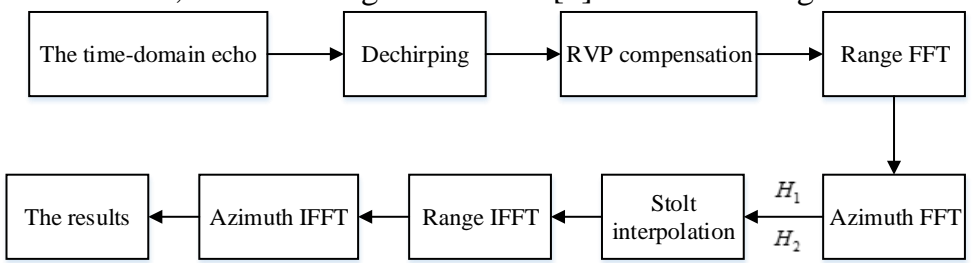

Fig. 1 The flow of RMA

\section{Algorithm Simulation and Test}

Next, we proceed RMA simulation and the simulation parameters are set as Table 1.

Table 1. The table of simulation parameters

\begin{tabular}{cc}
\hline Parameter & Value \\
\hline PRF & $1000[\mathrm{~Hz}]$ \\
Bandwidth & $100[\mathrm{MHz}]$ \\
Sampling rate & $200[\mathrm{MHz}]$ \\
$f_{c}$ & $34[\mathrm{GHz}]$ \\
$R_{s}$ & $1.8[\mathrm{~km}]$ \\
$\tau$ & $2[\mathrm{us}]$ \\
$V_{a}$ & $70[\mathrm{~km} / \mathrm{h}]$ \\
\hline
\end{tabular}

The point targets are set as Fig. 2. In the figure, there are nine point targets and the $X$ axis represents the centerline of the image scene. The square point is the centered goal. 


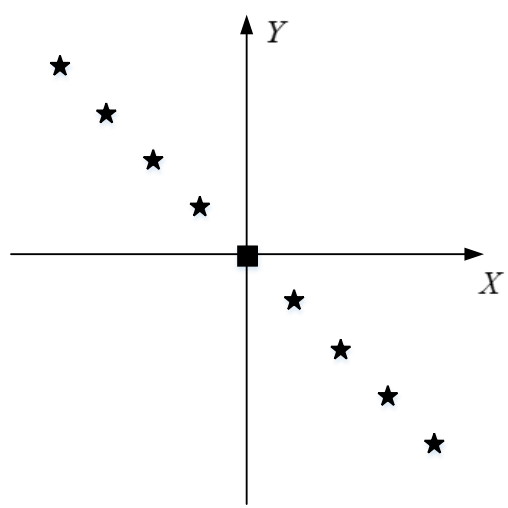

Fig. 2 The sketch of point targets distribution

The imaging results are shown in Fig. 3. Fig. 3-(a) is the results of no interpolation and Fig. 3-(b) is the results of interpolation. As can be seen from the figure, the two figures all can restore the target information, but the target of the interpolated one focus better. Fig. 3-(c) and Fig. 3-(d) are the azimuth sectional figure, we can see that the interpolated target has narrower main lobes and lower side lobes, so the target focuses better.

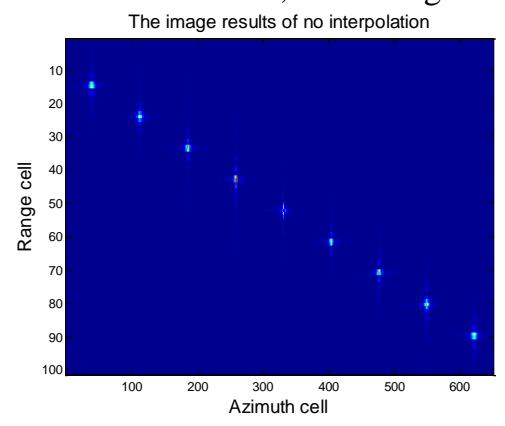

(a)

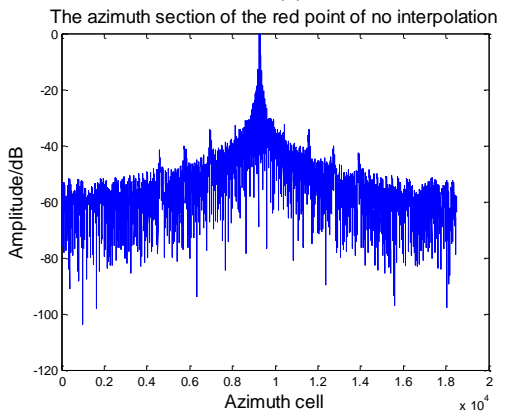

(b)

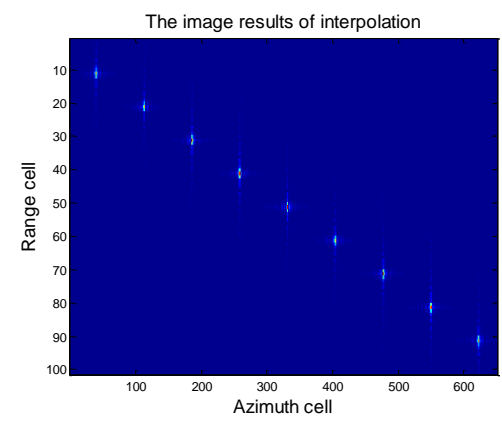

(b)

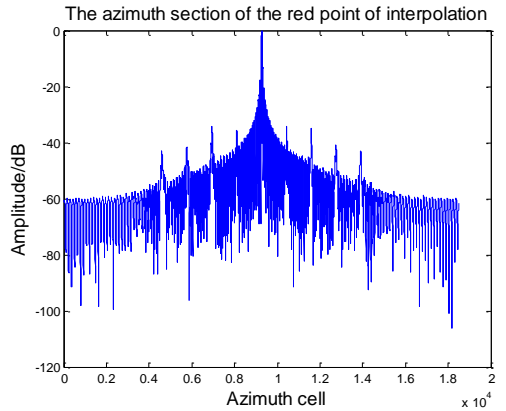

(d)

Fig. 3 The simulation results 
Figure. 4 is the contour image of the center target, we can see from the result that RMA reconstructs the target information better.

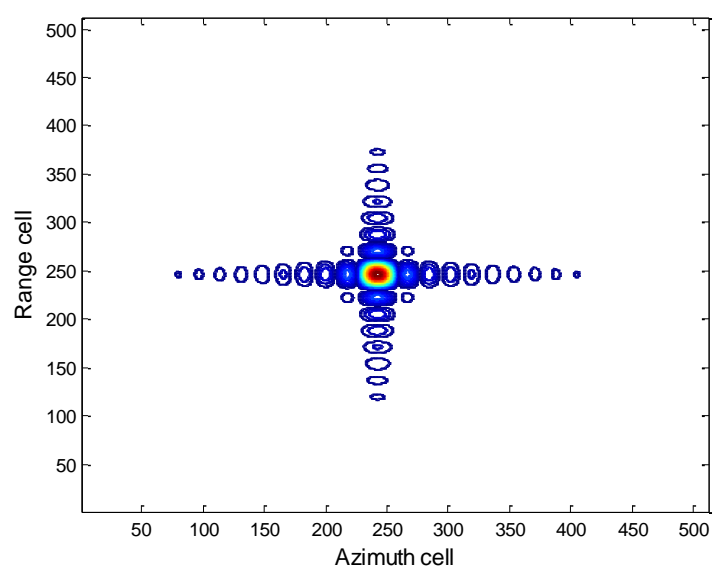

Fig. 4 The contour image of the center target

This algorithm was applied to the vehicle millimeter wave SAR system whose parameters were the same with the simulation parameters. The imaging experiment was proceeded in Sanchaji Bridge Changsha. We used the fullaperture data to image and stitched every child image in sequence. The image results obtained were as Fig. 5.

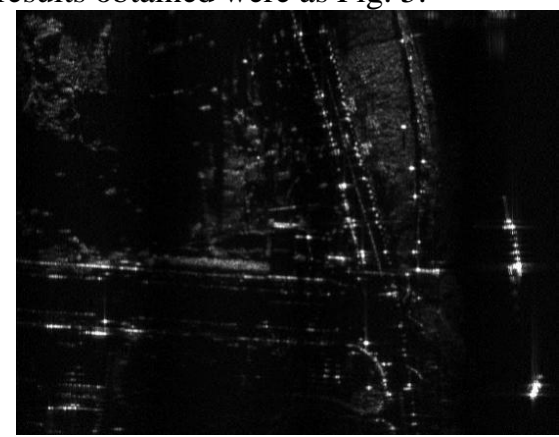

(a) The SAR image

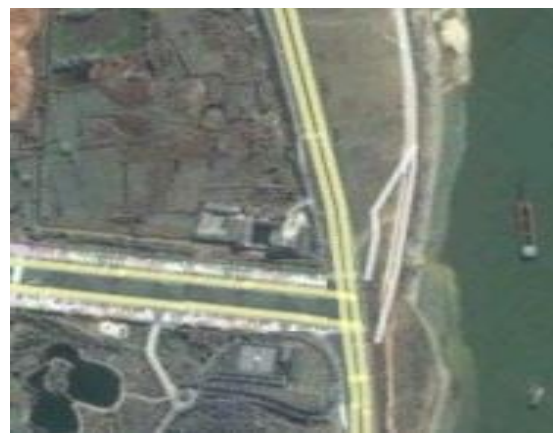

(b) The optical image

Fig. 5 The test results

Figure. 5-(a) is the SAR image obtained from the vehicle SAR system using RMA and Fig. 5-(b) is the optical image downloaded from the google map. We can see from the SAR image that it had a higher resolution and showed the main features of the image region and preferably reconstructed the object information. The SAR image corresponded to the characteristics of the vehicle platform: low grazing angle and little echo energy. So some features of the image region can't 
be fully rendered, but the main goals like road lights, riverbank, river boats in the image have a clear outline and a good focusing effect.

\section{Conclusion}

This paper presents a vehicle millimeter wave SAR system. Based on the platform characteristics, we choose RMA with the stolt interpolation to image. We proceeded the detailed analysis and simulation for the algorithm and we also carried through the imaging test. The results showed that the system is able to reconstruct the main information of objects in the imaging area. So, the system has a strong practical effect. Consequently, this vehicle millimeter wave SAR system has a very broad application prospect in the field of supervising the rivers and lakes and monitoring the resource and so on.

\section{References}

1. Z. Bao, M. D. Xing, T. Wang, Radar imaging technology, Publishing House of Electronics Industry, Beijing, 2009.

2. I. G. Cumming, F. H. Wong, Digital processing of synthetic aperture radar data: algorithms and implementation. Publishing House of Electronics Industry, Beijing, 2009.

3. Z. M. He, J. Zhu, B. Zhou, Research on Real-Time Signal Processing of the Missile-Borne SAR. Journal of Electronics \& Information Technology, 2008, vol. 30, pp. 1011-1013.

4. H. T. Ren, S. H. Wang, D. N. Liang, Research on Interpolating Methods for UWB-SAR Imagery. Journal of National University of Defense Technology, 1998, vol. 20, pp. 66-69.

5. F. Li, Study of $w$ - $k$ Processing Algorithm in SAR Data Processing, Aero Weaponry, 2006 vol. 6, pp. 10-12.

6. P. G. Liu, J. J. Mao, Radio Propagation and Antenna, National University of Defense Technology Press, Changsha, 2004.

7. D. Y. Zhu, Z. D. Zhu, S. H. Ye, Study and Practice of Imaging Signal Processing for Airborne SAR, Journal of Nanjing University of Aeronautics \& Astronautics. 